\title{
Entwicklung einer Glucosedehydrogenase-basierten Anode und deren Anwendung in einer Glucose/ $\mathrm{O}_{2}$-Biobrennstoffzelle
}

\author{
Christoph K. Tanne, Gero Göbel, Fred Lisdat
}

Zusammenfassung

Unter Verwendung von mehrwandigen Kohlenstoffnanoröhren wurde in dieser Studie eine neuartige Anode zum Einsatz in Biobrennstoffzellen entwickelt. Dazu wurde das rekombinante Enzym Pyrrolochinolinchinon(PQQ)abhängige Glucosedehydrogenase kovalent an eine aus PQQ bestehenden Zwischenschicht gekoppelt, welche zuvor an die Kohlenstoffnanoröhren adsorbiert war. Die Nanoröhren wurden aufgrund ihrer Thiolmodifikation chemisorptiv auf einer Goldelektrode gebunden. In glucosehaltiger Lösung konnte der Start eines katalytischen Stroms bei einem Potential von $-80 \mathrm{mV}$ vs. $\mathrm{Ag} / \mathrm{AgCl}$ ( $1 \mathrm{M}$ $\mathrm{KCl}$ ) beobachtet werden. Unter Substratsättigung wurden Stromdichten im Bereich von 170 bis $200 \mu \mathrm{A} / \mathrm{cm}^{2}$ gemessen. Dieses System basiert auf einem mediatorvermittelten Elektronentransfer.

Die entwickelte (PQQ)-GDH-MWCNT-Elektrode wurde mit einer MWCNT-modifizierten Elektrode kombiniert, bei der Bilirubinoxidase (BOD) als Biokatalysator fungiert. Daraus resultierte eine membranfreie Biobrennstoffzelle mit einem Gleichgewichtspotential von $600 \mathrm{mV}$ und Leistungsdichten im Bereich von 20-25 $\mu \mathrm{W} / \mathrm{cm}^{2}$.

\section{Abstract}

In this study a biofuel cell anode is developed on the basis of multi-walled carbon nanotubes (MWCNTs). Recombinant pyrroloquinoline quinone (PQQ) dependent glucose dehydrogenase is covalently coupled to a PQQ-layer which is adsorbed onto thiolmodified MWCNTs. The MWCNTs are chemisorbed to a gold electrode. In the presence of glucose a catalytic current starts at a potential of $-80 \mathrm{mV} v \mathrm{vs} . \mathrm{Ag} / \mathrm{AgCl}$, $1 \mathrm{M} \mathrm{KCl}$. Under substrate saturation current densities of 170 to $200 \mu \mathrm{A} / \mathrm{cm}^{2}$ can be achieved. The operation is based on mediated electron transfer of the enzyme.

This (PQQ)-GDH-MWCNT-electrode is combined with a MWCNT-modified electrode to which bilirubin oxidase (BOD) is covalently coupled. The resulting membrane-free biofuel cell has an open cell potential of $600 \mathrm{mV}$ and can achieve power densities in the range of $20-25 \mu \mathrm{W} / \mathrm{cm}^{2}$.

\section{Einleitung}

Aufgrund ihrer Fähigkeit, chemisch gespeicherte Energie effizient in elektrische Energie umzuwandeln, gewannen Brennstoffzellen in den letzten Jahren zunehmend öffentliches Interesse. Werden bei der Energieumwandlung biologische Katalysatoren verwendet, handelt es sich um so genannte Biobrennstoffzellen, welche bei Raumtemperatur und bei neutralem $\mathrm{pH}$ Wert betrieben werden können. Allerdings werden dabei eher nur geringe Leistungsdichten erbracht. Aus diesem Grund ist ihr Einsatz zwar auf Anwendungen mit geringen Leistungsanforderungen fixiert, jedoch können sie eine Reihe unterschiedlicher organischer Brennstoffe, wie Glucose, Fructose oder Ethanol, kon- vertieren. Wird der biokatalytische Umsatz eines Substrats durch Mikroorganismen bewerkstelligt, hat die Biobrennstoffzelle zwar eine verlängerte Lebensdauer, allerdings ist die Leistungsdichte zusätzlich gemindert. Hingegen sind auf reinen Enzymen basierende Brennstoffzellen zwar weniger stabil, erreichen jedoch höhere Energieausbeuten (Davis et al. 2007).

Für die Effektivität einer enzymatischen Biobrennstoffzelle ist ein schneller Elektronentransfer zwischen dem Enzym und der Elektrode ausschlaggebend. Dies kann durch Elektronentunneling erreicht werden. Daraus resultiert ein direkter Elektronentransfer (DET), ein schneller Mechanismus, der allerdings einen geringen Abstand zwischen dem aktiven Zentrum des Enzyms und der leitfähigen Oberfläche voraussetzt. Um dies zu 
erreichen kann das Enzym kovalent an die Elektrode gekoppelt werden (Willner et al. 2009). Meist kann jedoch nur ein Mediator-vermittelter Elektronentransfer (MET) die elektrische Kommunikation zwischen dem Protein und der Elektrode realisieren. Dazu können mobile Mediatoren wie NAD ${ }^{+}$und ABTS, immobilisierte Mediatoren auf Polymerbasis sowie leitfähige Polymere verwendet werden (Willner et al. 2009; Kim et al. 2006; Mao et al. 2003; Gallaway/Barton 2008). In polymeren Matrizen können große Mengen an Enzym immobilisiert werden, allerdings ist die Substratdiffusion limitiert. Die Fixierung kann durch die kovalente Kopplung der Biokatalysatoren an funktionelle Gruppen des Polymers oder einer festen Oberfläche stabilisiert werden.

In heutigen bioelektronischen Systemen kommen immer häufiger Materialien aus der Nanotechnologie zum Einsatz. Dabei handelt es sich um ein Wissenschaftsgebiet, in dem innerhalb der letzten Jahre viele Innovationen stattgefunden haben. Nanoskalierte Strukturen offerieren große Oberflächen, was die Möglichkeit hoher Enzymbeladungen erlaubt. Häufig werden Kohlenstoffnanoröhren genutzt (CNT, engl.: carbon nanotubes). Durch ihre einzigartigen mechanischen, strukturellen sowie elektrischen Eigenschaften sind sie sowohl als Elektrodenmaterial sowie als Elektrodenbeschichtung geeignet. Des Weiteren lassen sich Enzyme wirksam an sie koppeln, sofern die Nanoröhren funktionalisiert sind. Dadurch wird es möglich leistungsfähige Nano-Bio-Hybrid-Systeme im Miniaturformat zu konstruieren (Yang et al. 2007).

Innerhalb einer enzymatischen Biobrennstoffzelle werden für den Reduktionsprozess am kathodischen Element meist Laccasen, Peroxidasen oder Bilirubinoxidase (BOD) genutzt (Willner et al. 1998; Sakai et al. 2009; Wang et al. 2009; Kim et al. 2009). BOD wird oft verwendet, da sie in einem breitem $\mathrm{pH}$-Bereich mit hoher Aktivität arbeitet (Otsuka et al. 2007). Es ist bereits mehrfach publiziert worden, dass sich BOD leicht an Elektroden immobilisieren lässt, welche mit mehrwandigen Kohlenstoffnanoröhren (MWCNT) modifiziert sind, wodurch ein direkter Elektronentransfer erreicht werden konnte (Weigel et al. 2007; Schubert et al. 2009; Zheng et al. 2010).

Im anodischen Kompartiment werden für den Oxidationsprozess meist Enzyme, wie Glucoseoxidase, Glucosedehydrogenasen sowie Alkoholdehydrogenasen angewendet. Die lösliche und PQQ-abhängige Form der Glucosedehydrogenase, (PQQ)-GDH), ist für den Einsatz in Biobrennstoffzellen besonders interes- sant, da sie bei neutralem pH-Wert eine sehr hohe katalytische Aktivität aufweist und nicht von Sauerstoff abhängig ist (Ye et al. 1993; Yuhashi et al. 2005).

Der Elektrontransfer ist sowohl über den Mediatorvermittelten Weg als auch über den direkten Weg intensiv untersucht worden. Als Mediator werden häufig phenolische Verbindungen oder Cyanoferrate genutzt (Li et al. 2008; Lisdat et al. 1998). Es ist auch möglich (PQQ)-GDH an funktionalisierte einwandige Kohlenstoffnanoröhren zu binden und in diesem Fall einen direkten Elektronentransfer zu beobachten (Ivnitski et al. 2007). Weiterhin wurde publiziert, dass das Apoenzym dieser GDH-Variante an kovalent gebundenem PQQ rekonstituiert werden kann (Zayats et al. 2005).

Die vorliegende Arbeit beschreibt die Entwicklung einer neuen Proteinanode, welche potentiell in Biobrennstoffzellen Einsatz finden kann. Das Elektrodensystem basiert auf thiolmodifizierten, mehrwandigen Kohlenstoffnanoröhren, welche chemisorptiv auf einer Goldelektrode fixiert sind. Die PQQ-abhängige GDH, welche die Oxidation von Glucose katalysiert, wurde als Biokatalysator gewählt. Des Weiteren wird eine Biobrennstoffzelle vorgestellt in welcher die (PQQ)-GDH-MWCNT-Anode mit einer BOD-MWCNTKathode kombiniert wird. Beide machen von der hohen bioelektrokatalytischen Aktivität der Enzyme an den goldgebundenen MWCNTs Gebrauch. Die grundlegenden Eigenschaften einer solchen membranfreien Biobrennstoffzelle sind bestimmt worden.

\section{Material und Methoden}

\section{Reagenzien}

Mehrwandige Kohlenstoffnanoröhren (MWCNT) wurden von NANOCYL S.A. (Belgien) geliefert. 2-(4-(2-Hydroxyethyl)-1-piperazinyl)-ethan-sulfonsäure (HEPES) und 2-(N-Morpholino)ethan-sulfonsäure sind von AppliChem GmbH (Deutschland) bezogen worden. Zitronensäure wurde von Merck KGaA (Deutschland), Pyrrolochinolinchinon (PQQ), D-Glucose, 1-Ethyl-3-(3-dimethylaminopropyl) carbodiimid (EDC), N-hydroxysuccinimid (NHS), Sulfo-N-succinimidyl-4-maleimidobutyrat (S-GMBS) und Bilirubinoxidase (BOD) aus Myrothecium verrucaria wurden von Sigma Aldrich Chemie GmbH (Deutschland) bezogen. Di-Natriumhydrogenphosphat und Kalziumchlorid wurden von Roth GmbH + Co KG (Deutschland) geliefert. Ethanol und Natriumacetat-Trihydrat wurden 
von neoLab GmbH (Deutschland) bezogen. Siliziumcarbidpapier war von Dieter Schmid Feine Werkzeuge GmbH (Deutschland). Sämtliche wässrige Lösungen sind mit gereinigtem (deionisiertem) Wasser aus dem Aufbereitungssystem »Ultra Clear direct« der Firma SG Water (Deutschland) hergestellt worden. Lösliche PQQ-abhängige Glucosedehydrogenase [(PQQ)-GDH] (MW=86.116 kDa) wurde als Apoenzym von Roche Diagnostics GmbH (Deutschland) großzügig zur Verfügung gestellt. Das Apoenzym wurde rekombinant aus Escherichia coli gewonnen, wobei die Gene für die Expression periplasmatischer Glucosedehydrogenase aus dem Bakterium Acinetobacter calcoaceticus stammen. Die aktive Form des Enzyms wurde in einer Konzentration von $1 \mathrm{mg} / \mathrm{ml}$ apo-GDH in $50 \mathrm{mM}$ MES-Puffer bei pH 6,5 (mit KOH justiert) mit $1 \mathrm{mM} \mathrm{CaCl}_{2}$ und $150 \mu \mathrm{M}$ PQQ für 3 h bei Raumtemperatur rekonstituiert.

\section{Präparation der Enzymelektroden}

Aus einer MWCNT-Stammlösung (10 mg/ml in 5 mM Zitrat-Phosphatpuffer, $\mathrm{pH} 7$ ) wurde ein Teil mit neun Teilen 96\%-igem Ethanol vermischt und 8 bis 16 Stunden mit Ultraschall behandelt.

Goldelektroden wurden nass auf Siliziumcarbidpapier verschiedener Korngrößen (1.200, 2.000 und 3.000) geschliffen. Zweimal 4 pl MWCNT-Ethanolsuspension wurden daraufhin nacheinander auf die Elektrodenfläche gegeben und getrocknet.

Für die Kopplung der (PQQ)-GDH wurde eine organische Zwischenschicht aus PQQ-Molekülen aus Lösungen mit 0,5 mM bzw. 15 mM PQQ auf den MWCNTs adsorbiert. Die Carboxylgruppen der abgelagerten PQQ-Moleküle wurden mit $100 \mu \mathrm{l}$ von $50 \mathrm{mM}$ EDC und 50 mM NHS über 15 Minuten chemisch aktiviert. Danach wurden $20 \mu \mathrm{l}$ der $1 \mathrm{mg} / \mathrm{ml}$ Lösung rekonstituierter (PQQ)-GDH für eine Stunde auf die aktivierte Elektrodenfläche gegeben. Danach wurde die Elektrode dreimal mit 300 pl 50 mM MES-Puffer gewaschen.

Um die BOD an die MWCNT-modifizierte Goldelektrode zu koppeln, wurden die Elektroden nach dem Auftragen der Kohlenstoffnanoröhren für 30 Minuten in $8 \mathrm{mM}$ Sulfo-GMBS-Lösung in $25 \mathrm{mM}$ Natriumphosphatpuffer $(\mathrm{pH} \mathrm{7,5)} \mathrm{inkubiert.} \mathrm{Danach} \mathrm{wurden} \mathrm{sofort}$ $20 \mu \mathrm{l}$ von $10 \mu \mathrm{M}$ BOD-Lösung (BOD in $5 \mathrm{mM}$ ZitratPhosphat-Puffer) für eine Stunde auf die MWCNT-Fläche der Elektrode aufgetragen. Unspezifisch gebundenes Enzym und weitere Verunreinigungen wurden im Anschluss dreifach mit Zitrat-Phosphat-Puffer ( $\mathrm{pH} 7$ ) abgewaschen.

\section{Elektrochemische Charakterisierung}

Für die elektrochemische Charakterisierung der beiden einzelnen Elektroden wurde die Dreielektrodenanordnung verwendet. Bei der Arbeitselektrode handelte es sich um eine Goldstabelektrode (geometrische Fläche: 2,01 $\mathrm{mm}^{2}$ ) von Bioanalytical Systems Ltd. (Großbritannien). Als Referenzelektrode dienten eine Ag/AgClElektrode (1 $\mathrm{M} \mathrm{KCl)} \mathrm{von} \mathrm{Microelectrodes} \mathrm{Inc.} \mathrm{(USA)} \mathrm{und}$ ein Platindraht als Gegenelektrode.

Voltammetrische Untersuchungen der (PQQ)-GDHMWCNT-Elektrode wurden am Autolab PGSTAT12 (Metrohm) durchgeführt. Sämtliche elektrochemischen Messungen zur Untersuchung der Biobrennstoffzellenanwendung wurden mit dem Potentiostat Gamry Reference 600 (USA) vorgenommen.

Das Potential des Schaltkreises (über- und unterlastig) wurde im OCP-Modus mit dem Potentiostat mit der (PQQ)-GDH-MWCNT-Elektrode als Arbeitselektrode und der BOD-MWCNT-Elektrode in der Referenzelektrodenposition gemessen. Mit der (PQQ)-GDHMWCNT-Elektrode als Arbeitselektrode und mit der BOD-MWCNT-Elektrode unter simultaner Schaltung in der Referenz- und Gegenelektrodenposition wurden galvanodynamische Messungen ermöglicht.

Für die Bestimmung des Gleichgewichtspotentials wurde die Zellspannung mit und ohne Stromfluss und für galvanodynamische Charakterisierungen 20 millimolare Glucoselösung in luftgesättigtem 50 millimolarem Puffer mit $1 \mathrm{mM} \mathrm{CaCl}_{2}$ (pH 6,5) verwendet. Eine Scanrate von $2 \mathrm{nA} / \mathrm{s}$ wurde für die galvanodynamischen Messungen der enzymatischen Biobrennstoffzelle genutzt.

\section{Ergebnisse und Diskussion}

Um sich die hohe bioelektrokatalytische Aktivität der BOD-MWCNT-Elektrode in einer Biobrennstoffzelle als Kathode zu Nutze zu machen, wurde eine neue Anode entwickelt, welche auf einem sehr ähnlichen Elektrodendesign basiert. Hierbei wurde eine elektrochemische Kommunikation der (PQQ)-GDH mit der MWCNT-modifizierten Elektrode realisiert. Diese Proteinelektrode wurde im Hinblick auf ihre Fähigkeit zur Glucoseoxidation untersucht. Im Anschluss wurde diese Elektrode mit der BOD-MWCNT-Kathode kombiniert und die Leistung der daraus resultierenden enzymatischen Biobrennstoffzelle untersucht. 
Realisierung von (PQQ)-GDH-Elektrodenkontakten durch MWCNTs

Um einen Kontakt zwischen dem Enzym und der Elektrode herzustellen, wurde zunächst versucht (PQQ)GDH aus einer gepufferten Lösung $(\mathrm{pH}$ 6,5) an den mehrwandigen Kohlenstoffnanoröhren $\mathrm{zu}$ adsorbieren. Die voltammetrische Untersuchung solcher Elektroden zeigte jedoch, dass in Glucose-haltiger Lösung kein DET beobachtet werden konnte. Daher wurde ein alternativer Weg gesucht. Es ist bekannt, dass sich PQQ leicht aufgrund seiner aromatischen Struktur an CNTs adsorbieren lässt, wodurch eine organische Zwischenschicht erzeugt werden kann (Goebel/Lisdat 2008). Dies vereinfacht auch die kovalente Kopplung von Enzymen, welche durch die chemische Aktivierung der PQQ-Carboxylgruppen mit Carbodiimid erreicht wird. Abb. 1. zeigt den schematischen Aufbau einer solchen Proteinelektrode.

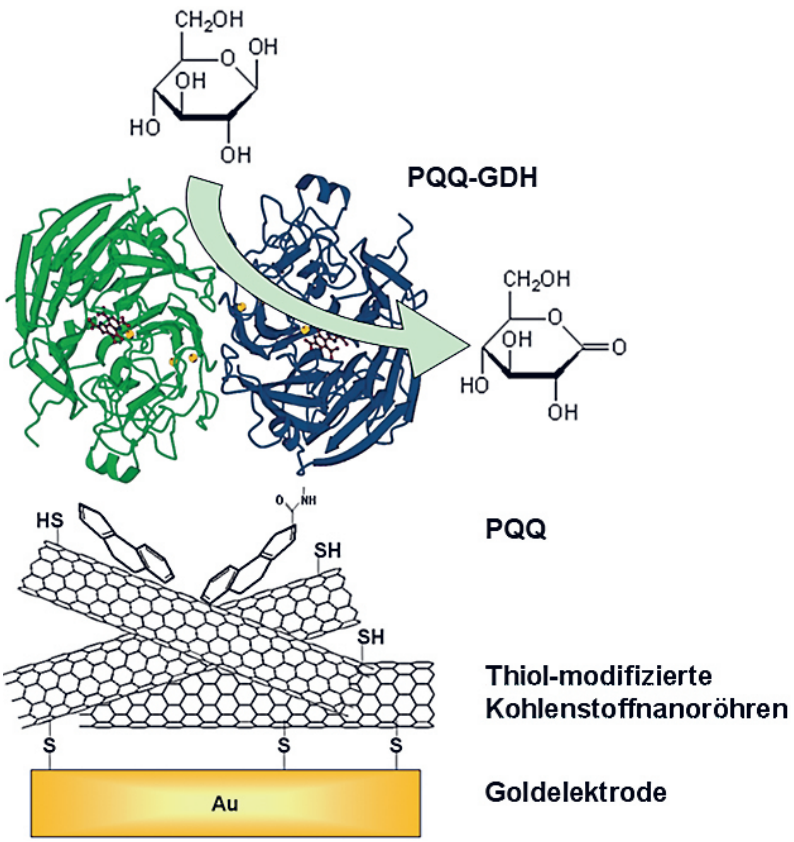

Abb. 1: Schematische Skizze des (PQQ)-GDH-Elektrodendesigns

Um die Realisierbarkeit des geplanten Aufbaus zu testen, wurde PQQ aus verdünnter Lösung adsorbiert und (PQQ)-GDH kovalent daran gekoppelt. Die so konstruierten Elektroden wurden anschließend über LSVMessungen (Linear Sweep Voltammetry) elektrochemisch untersucht. Wie in Abb. 2a ersichtlich, erscheint bei Messungen in Glucose-freier Lösung ein Oxidationspeak nahe dem formalen Redoxpotential des PQQ (-80 $\mathrm{mV}$ vs. Ag/AgCl, $1 \mathrm{M} \mathrm{KCl}$ ).

In weiteren voltammetrischen Messungen konnte ein erhöhter Strom in Anwesenheit von Glucose beob-
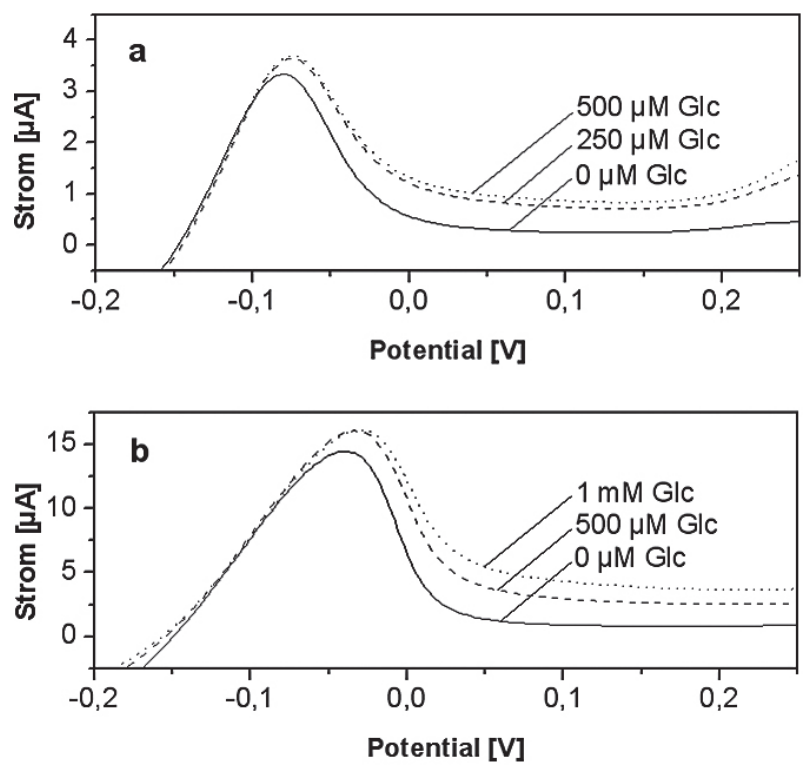

Abb. 2: Voltammetrische Untersuchung der (PQQ)-GDH-MWCNTElektrode: a) PQQ aus $500 \mu \mathrm{M}$ - Lösung adsorbiert. b) PQQ aus $15 \mathrm{mM}$ Lösung adsorbiert (Messbedingungen: $10 \mathrm{mV} / \mathrm{s}$, in $50 \mathrm{mM}$ MES, $1 \mathrm{mM} \mathrm{CaCl}_{2}, \mathrm{pH} \mathrm{6,5}$, vs. $\mathrm{Ag} / \mathrm{AgCl}, 1 \mathrm{M} \mathrm{KCl}$ ).

achtet werden, welcher mit der Glucosekonzentration anstieg. PQQ interagiert mit der PQQ-abhängigen GDH in natürlicher Weise, da es auch der eigentliche Cofaktor dieses Enzyms ist.

Der Glucose-abhängige Stromanstieg wurde erst dann beobachtet, wenn alle PQQ-Moleküle oxidiert waren. Dies impliziert, dass das an den MWCNTs adsorbierte PQQ als Mediator in diesem System fungiert, anders als bei einer BOD-PQQ-MWCNT-Kathode, wo es als Promoter agieren konnte (Goebel/Lisdat 2008).

In der ersten experimentellen Reihe wurde PQQ aus eher niedrig konzentrierter Lösung $(0,5 \mathrm{mM})$ adsorbiert. Dieser Ansatz war zwar erfolgreich, es wurden jedoch meist nur geringe Ströme in Anwesenheit von Glucose erzielt. Die Oberflächenkonzentration des PQQ betrug dabei $5 \pm 2 \mathrm{nmol} / \mathrm{cm}^{2}$ (ausgehend von der geometrischen Elektrodenfläche). Zur Erhöhung der katalytischen Aktivität der Elektrode wurden höhere PQQOberflächenkonzentrationen untersucht. Um diese zu erreichen, wurde die MWCNT-beschichtete Elektrode vor der Enzymkopplung mit 15 mM PQQ-Lösung behandelt. Abb. 2b zeigt das voltammetrische Verhalten einer solchen Elektrode. Die zu beobachtende Vergrößerung der Oxidationspeakfläche deutet auf größere Mengen an CNT-fixiertem PQQ. Aus den Ergebnissen mehrerer Messungen wurde eine durchschnittliche PQQ-Oberflächenkonzentration von $26 \pm 2 \mathrm{nmol} / \mathrm{cm}^{2}$ berechnet (ausgehend von der geometrischen Elektrodenfläche). Die dadurch erreichte Verbesserung im 

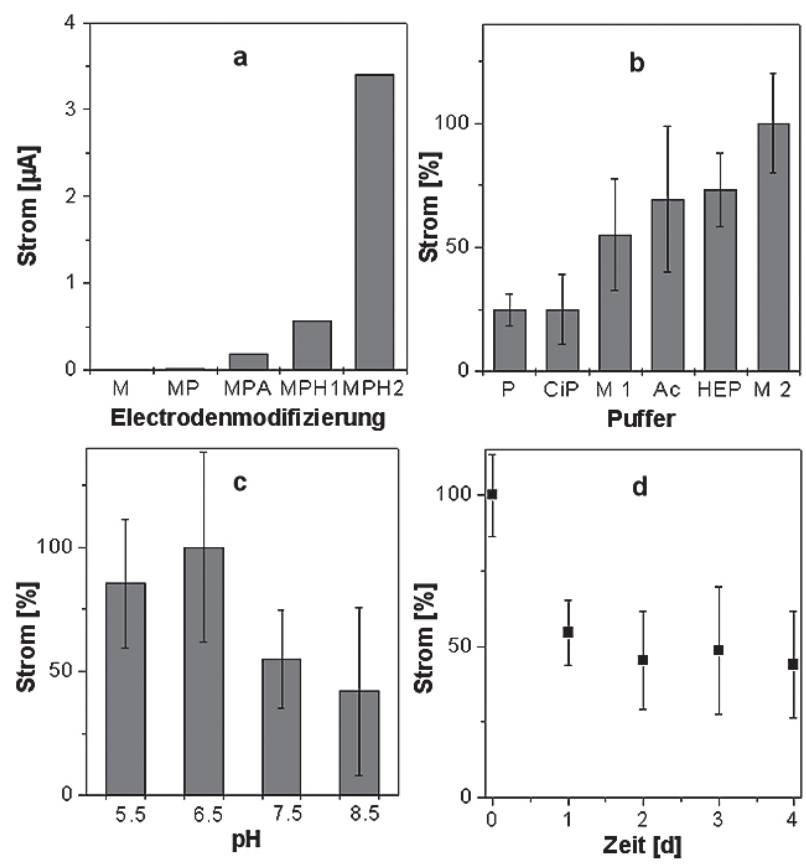

Abb. 3: Voltammetrische Stromantwort der (PQQ)-GDH-MWCNTElektrode bei $1 \mathrm{mM}$ Glucose und 0,1 V vs. $\mathrm{Ag} / \mathrm{AgCl}(1 \mathrm{M} \mathrm{KCl}, 10 \mathrm{mV} / \mathrm{s})$ a) In Abhängigkeit der Elektrodenmodifizierung: M: MWCNT-Elektrode; MP: MWCNT-PQQ-Elektrode; MPA: MWCNT/PQQ-Elektrode mit apo-GDH; MPH1: MWCNT/PQQ-Elektrode mit holo-GDH; MPH2: MWCNT/PQQElektrode mit holo-GDH. (PQQ wurde aus einer 0,5 mM Lösung an die MWCNT adsorbiert, nur für MPH2 wurde PQQ aus einer 15 mM PQQ-Lösung verwendet; Messbedingungen: $50 \mathrm{mM} \mathrm{MES,} 1 \mathrm{mM} \mathrm{CaCl}_{2}, \mathrm{pH}$ 6,5) b) In Abhängigkeit der Pufferzusammensetzung: P: $50 \mathrm{mM}$ PhosphatPuffer, pH 6,5; CiP: 50 mM Citrat-Phosphat-Puffer, pH 6,5; M 1: 50 mM MES-Puffer, pH 6,5; Ac: 50 mM Acetat-Puffer, pH 6,5; HEP: 50 mM HEPESPuffer, pH 6,5 ; M 2: 50 mM MES-Puffer, 1 mM CaCl $2, p H$ 6,5

c) In Abhängigkeit des $\mathrm{pH}$-Wertes (Messbedingungen: $50 \mathrm{mM} \mathrm{MES}$, $1 \mathrm{mM} \mathrm{CaCl}_{2}$ )

d) Langzeitstabilität der (PQQ)-GDH-MWCNT-Elekroden (Messbedingungen: LSV, $10 \mathrm{mV} / \mathrm{s}, 50 \mathrm{mM} \mathrm{MES,} 1 \mathrm{mM} \mathrm{CaCl}$, $\mathrm{pH}$ 6,5; Lagerungsbedingungen: $50 \mathrm{mM} \mathrm{MES}, 1 \mathrm{mM} \mathrm{CaCl}_{2}, 100 \mu \mathrm{M}$ PQQ, pH 6,5, $4^{\circ} \mathrm{C}$ ) Die Abbildungen b) bis d) zeigen die relative Änderung des Stromsignals im Vergleich zur stärksten Stromantwort. Die Fehlerbalken resultieren aus 3 unabhängigen Messungen.

Elektronentransfer zwischen dem Enzym und der Elektrode zeigte sich in erhöhten Stromdichten. Unter Verwendung der gleichen Enzymkopplungsstrategie wie zuvor, konnten in Anwesenheit von Glucose ebenfalls höhere Ströme beobachtet werden $\left(170-200 \mu \mathrm{A} / \mathrm{cm}^{2}\right)$. Dies deutet darauf hin, dass eine hohe Oberflächenkonzentration an PQQ günstig für die Kontaktierung vieler (PQQ)-GDH-Moleküle ist.

Zur Validierung der Glucoseoxidation im Falle immobilisierter (PQQ)-GDH wurden zusätzlich Negativkontrollen durchgeführt. Waren die Elektroden nur mit MWCNTs oder nur mit MWCNTs und adsorbiertem PQQ modifiziert, so wurde kein katalytischer Strom detektiert (Abb. 3a). Allerdings muss erwähnt werden, dass sich geringe katalytische Ströme beobachten ließen, wenn an das adsorbierte PQQ zusätzlich das Apoenzym der GDH gekoppelt wurde, was mit einer wahrscheinlichen Rekonstitution einiger der Enzymmoleküle zur Holoform erklärbar ist.

\section{Sensorische Funktion der (PQQ)-GDH-MWCNT-}

\section{Elektrode}

Mit Hilfe amperometrischer Messungen, wurde die optimierte Proteinelektrode auf ihre Sensitivität untersucht. Abb. 4 zeigt, dass der stationäre Strom von der Glucosekonzentration abhängig war. Im Bereich von 5 bis $500 \mu \mathrm{M}$ verhielt sich der Strom linear ansteigend mit der Glucosekonzentration und ging im Bereich von $500 \mu \mathrm{M}$ bis $5 \mathrm{mM}$ in einen Sättigungsbereich über. Daraus ließ sich ein Maximalstrom von $(9,9 \mu \mathrm{A})$ berechnen, bei dem das Enzym mit maximaler Umsatzrate arbeitete. Messungen mit hoher Glucosekonzentration (10 mM) bestätigten diese Berechnung.

Zusätzlich wurde der apparente $\mathrm{K}_{\mathrm{M}}$-Wert mit $160 \mu \mathrm{M}$ berechnet. In der Literatur finden sich Werte für GDH in Lösung von 0,5 bis 22 mM (Laurinavièius et al. 2003; Dokter et al. 1986). Für immobilisierte (PQQ)-GDH sind die Werte der apparenten Michaelis-Menten-Konstante im gleichen Bereich (Razumiene et al. 2000; Li et al. 2008). Der hier berechnete Wert deutet darauf hin, dass die Substratsättigung bereits bei eher geringen Konzentrationen erreicht wird, was wahrscheinlich durch starke Wechselwirkungen zwischen dem Protein und den mehrwandigen Kohlenstoffnanoröhren verursacht wird, die den Zugang des Substrats Glucose zum Enzym erschweren.

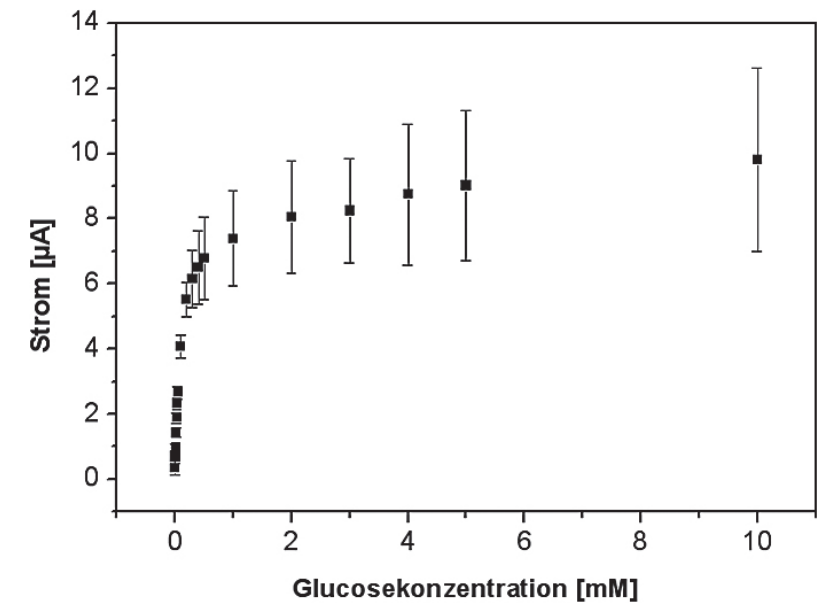

Abb. 4) Glucosesensitivität der (PQQ)-GDH-MWCNT-Elektrode (Amperometrische Messung bei $0,1 \mathrm{~V}$ vs. Ag/AgCl, $1 \mathrm{M} \mathrm{KCl}$ in $50 \mathrm{mM} \mathrm{MES}$, $1 \mathrm{mM} \mathrm{CaCl}_{2}, \mathrm{pH}$ 6,5) Die Fehlerbalken resultieren von 3 verschiedenen Elektroden und zeigen die Reproduzierbarkeit der Präparation 
Puffereffekte und Langzeitstabilität

Um die Auswirkungen verschiedener Pufferzusammensetzungen auf die Stromantwort des Elektrodensystems zu untersuchen, wurden verschiedene Lösungen getestet. Zur Evaluierung wurde eine Glucosekonzentration von $1 \mathrm{mM}$ verwendet und der Strom bei $+100 \mathrm{mV}$ beobachtet. Abb. 3b stellt die Ergebnisse dieser Versuche dar. Es war ersichtlich, dass in Anwesenheit von Phosphationen die enzymatische Aktivität an der Elektrodenoberfläche deutlich vermindert war. Wahrscheinlich verschiebt die Bildung von Kalziumphosphat das Gleichgewicht zwischen Enzym gebundenen $\mathrm{Ca}^{2+}$-Ionen und $\mathrm{Ca}^{2+}$-Ionen in der Lösung (verbunden mit einer verminderten PQQ-Bindung im Enzym). In HEPES- und Acetat-Puffer war die Stromantwort nur etwas kleiner als in MES-Puffer. Es ist offensichtlich, dass $\mathrm{Ca}^{2+}$-haltige Puffer einen positiven Einfluss auf das Stromsignal haben, so wie es auch schon für das in Lösung befindliche Enzym gezeigt wurde.

Um den Einfluss des pH-Wertes auf die bioelektrokatalytische Aktivität der Elektrode zu untersuchen, wurden verschiedene Elektroden in unterschiedlich gepufferten MES-Lösungen getestet. Für jeden pH-Wert
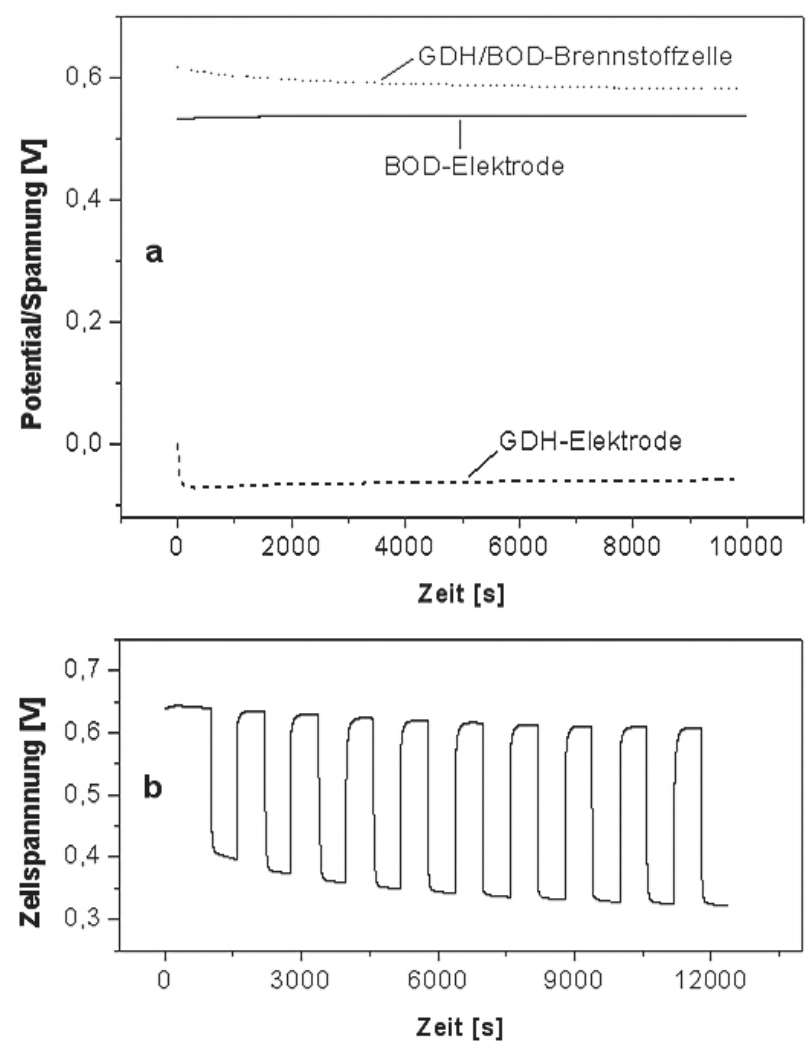

Abb. 5a) Stabilität des OCP (Messbedingungen: 50 mM MES, 1 mM $\mathrm{CaCl}_{2}$, pH 6,5 vs. Ag/AgCl, $1 \mathrm{M} \mathrm{KCl}$ ) b) Stabilität der Zellspannung der Biobrennstoffzelle mit und ohne Last (500 k $\Omega$-Widerstand); (Messbedingungen: $50 \mathrm{mM}$ MES, $1 \mathrm{mM} \mathrm{CaCl}$, $\mathrm{pH} \mathrm{6,5}$, vs. $\mathrm{Ag} / \mathrm{AgCl}, 1 \mathrm{M} \mathrm{KCl}$ ) wurden drei separate Elektroden untersucht. Abb. 3c zeigt die gemessenen Ströme bei $1 \mathrm{mM}$ Glucose bei einem Potential von $+100 \mathrm{mV}$. Dabei wurde der höchste Strom bei $\mathrm{pH}$ 6,5 beobachtet. Allerdings arbeitete das System auch in schwach saurem oder leicht basischem Milieu. Für die Aktivität des bioelektrokatalytischen Systems ist nicht nur das pH-Optimum des Enzyms entscheidend, sondern auch die Stärke der Wechselwirkung zwischen dem Protein und den PQQ-modifizierten MWCNTs. In der Literatur wird das pH-Optimum immobilisierter (PQQ)-GDH mit pH 6,0 angegeben (Li et al. 2008). Daraus kann geschlussfolgert werden, dass die enzymatische Aktivität wahrscheinlich größeren Einfluss auf die Leistung der Elektrode hat als das Elektrodendesign an sich.

Um Langzeiteffekte während der Lagerung der (PQQ)-GDH-MWCNT-Elektrode zu untersuchen, wurden mehrere Elektroden täglich voltammetrisch getestet (Lagerung bei $4{ }^{\circ} \mathrm{C}$ in Glucose-freier Lösung). Abb. 3d zeigt das Stabilitätsverhalten solcher Elektroden über mehrere Tage. Es ist offensichtlich, dass die Stromantwort innerhalb von einem Tag deutlich sinkt, sich jedoch im Weiteren stabil verhält.

\section{Anwendung in einer Biobrennstoffzelle}

Die (PQQ)-GDH-MWCNT-Elektrode wurde mit der BOD-MWCNT-Elektrode $\mathrm{zu}$ einer enzymatischen Biobrennstoffzelle kombiniert, da beide Elektroden vergleichbare bioelektrokatalytische Aktivitäten aufweisen. Die Elektroden erreichen Stromdichten im Bereich hunderter $\mu \mathrm{A} / \mathrm{cm}^{2}$ bei neutralem $\mathrm{pH}$-Wert und sind durch Enzym-MWCNT-Kopplung herstellbar. Durch die Kombination dieser beiden Proteinelektroden konnte ein membranfreies System aufgebaut werden, da die Substrate der jeweiligen Enzyme nicht mit den Elektrodenreaktionen des anderen Enzyms interferieren. Der Elektronenfluss verläuft von der Glucose über die GDH, PQQ zu den MWCNTs und dann über den externen Stromkreis zur Kathode, wo die Elektronen von der BOD auf den finalen Elektronenakzeptor Sauerstoff übertragen werden und dieser zu Wasser reduziert wird. Obgleich beide Elektroden auf Protein-MWCNTInteraktionen basieren, nutzt die Anode einen Mediator-vermittelten Elektronentransfer und die Kathode hingegen den direkten Elektronentransfer (Schubert et al. 2009).

Messungen des Gleichgewichtspotentials wurden für jeden Elektrodentyp separat und dann mit der vollständigen Biobrennstoffzelle durchgeführt. Das 
Potential der BOD-MWCNT-Elektrode in Sauerstoffgesättigter Lösung wurde mit $520 \mathrm{mV}$ vs. Ag/AgCl (1 M $\mathrm{KCl})$ bestimmt. Dieser Wert ist nah an dem thermodynamischen Redoxpotential der Sauerstoffreduktion bei neutralem pH-Wert (Stryer 1995). Für die (PQQ)-GDHMWCNT-Elektrode in Glucose-haltiger Lösung wurde ein Potential von -80 mV vs. Ag/Agcl (1 M KCl) ermittelt, was nahezu mit dem Redoxpotential des Enzyms übereinstimmt, wenn es in einem Chitosan-Kohlenstoffnanoröhren-Film auf einer GCE-Elektrode immobilisiert wurde (Sun et al. 2008). Dieser Wert ist etwas höher als das Redoxpotential des freien PQQ, welches bei -105 mV liegt (Katz et. al 1994).

Beide Elektroden wiesen über mehrere Stunden ein eher stabiles Gleichgewichtspotential auf, wobei sich das Potential der (PQQ)-GDH-MWCNT-Elektroden in den ersten 45 Minuten leicht verminderte. Für die gesamte Biobrennstoffzelle wurde ein Gleichgewichtspotential von $600 \mathrm{mV}$ gemessen. Auch hier zeigte sich ein stabiles Verhalten des Gleichgewichtspotentials über mehrere Stunden, wie Abb. 5a illustriert.

Der Verlust im Zellpotential kann durch den bei der (PQQ)-GDH-MWCNT-Elektrode beobachteten Abfall des Gleichgewichtspotentials erklärt werden. Offensichtlich ist der direkte Elektronentransfer von der BOD auf die Nanoröhren stabiler als der in einem System mit Mediator-vermitteltem Elektronentransfer, wie es bei der GDH-Elektrode der Fall ist.

Um die Stabilität der aufgebauten Biobrennstoffzelle während und nach einer Belastung zu bewerten, wurde in den Stromkreislauf ein $500 \mathrm{k} \Omega$-Widerstand eingebaut. Die beobachteten Ergebnisse (Abb. 5b) zeigten, dass sich die gemessene Terminalspannung nach einer gewissen Einlaufphase für beide Stromsituationen stabilisierte. Während der Versuche minderte sich die Spannung bei elektrischer Belastung um 75 mV, während das Gleichgewichtspotential nur um $30 \mathrm{mV}$ unter dem Initialwert lag.

Um eine Biobrennstoffzelle zu charakterisieren, sind die beiden Parameter Spannung und Leistungsdichte in Abhängigkeit von der Stromdichte entscheidend. Aus den voltammetrischen Untersuchungen ließen sich für die beiden einzelnen Elektroden Leistungskurven erstellen (Abb. 6a). In dieser Analyse stellte sich heraus, dass in der Kombination der beiden Elektrodensysteme die (PQQ)-GDH-MWCNT-Elektrode den limitierenden Faktor darstellt. Der sich aus den voltammetrischen Messungen ergebende Strom wurde durch eine angelegte Spannung verursacht, was eigentlich das gegen-
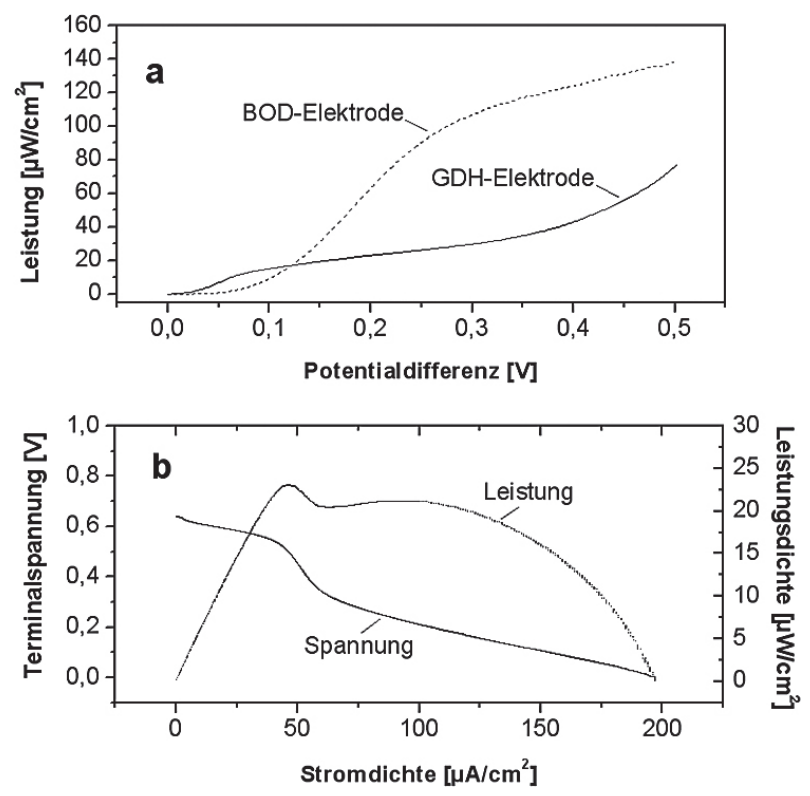

Abb. 6: a) Leistungskurven der einzelnen Enzymelektroden, berechnet aus den Daten der Linearen Sweepvoltammetrie (Scanrate $10 \mathrm{mV} / \mathrm{s}$ ) in luftgesättigter $20 \mathrm{mM}$ Glucoselösung. (Die X-Achse stellt die Differenz der angelegten Spannung zum OCP der jeweiligen Elektrode dar.) b) Leistungs- und Spannungskurve der gesamten Biobrennstoffzelle, erhalten aus galvanodynamischen Messungen bei einer Scanrate von $5 \mathrm{nA} / \mathrm{s}$. (Messbedingungen: luftgesättigter $50 \mathrm{mM} \mathrm{MES-Puffer,} 20 \mathrm{mM}$ glucose, $1 \mathrm{mM} \mathrm{CaCl}_{2}$, $\mathrm{pH} 6,5$ )

teilige Prinzip einer Brennstoffzelle darstellt. Daher wurde die Terminalspannung in Abhängigkeit von der elektrischen Belastung untersucht. Zu diesem Zweck wurden galvanodynamische Messungen bei geringen Stromvorschubgeschwindigkeiten durchgeführt, woraus sich der typische Kurvenverlauf der Leistungsdichte berechnen ließ.

Abb. 6b zeigt das Leistungsdiagramm einer Biobrennstoffzelle, in welcher beide MWCNT-basierenden Enzymelektroden vereint waren. Es wurde eine maximale Leistungsdichte im Bereich von 20-25 $\mu \mathrm{W} / \mathrm{cm}^{2}$ erreicht. Dabei zeigte sich bis zu einer Stromdichte von $45 \mu \mathrm{A} /$ $\mathrm{cm}^{2}$ ein eher nur geringer Spannungsabfall, wodurch die Leistung schnell bei kleinen Stromdichten stieg. Ab diesem Punkt bis zu einem Stromfluss von $70 \mu \mathrm{A} / \mathrm{cm}^{2}$ fand ein schneller Terminalspannungsabfall statt und stabilisierte sich danach bei einer geringeren Spannung bei fast konstanter Rate. Die Leistungsdichte der hier vorgestellten Biobrennstoffzelle übersteigt die Werte wie sie für Osmium-Polymer- oder Polyacrylamidbasierte enzymatische Biobrennstoffzellen ermittelt wurden (Stoica et al. 2009; Katz/Willner 2003). Die erzielte Leistungsdichte ist hingegen vergleichbar mit solchen Biobrennstoffzellen, die auf dem Einsatz von Polypyrrol oder einem anderen Kohlenstoffmaterial für die 
Realisierung des Enzym-Elektroden-Kontakts beruhen (Zhao et al. 2009; Gao et al. 2007). Jedoch wurde auch von enzymatischen Biobrennstoffzellen berichtet, die höhere Leistungsdichten erzielen. Diese nutzten zum Beispiel Polythiophen-Derivate oder dreidimensionale Goldnanopartikelanordnungen, welche den elektrischen Kontakt zwischen Elektrode und dem Enzym herstellten (Kuwahara et al. 2009; Murata et al. 2009).

\section{Zusammenfassung}

Die Etablierung einer neuen Proteinanode, welche auf dem Mediator-vermittelten Elektronentransfer zwischen hoch aktiver (PQQ)-GDH (3.000 U/mg) und einer MWCNT-modifizierten Goldelektrode basiert, wurde erfolgreich umgesetzt. Dabei übernahm an MWCNT adsorbiertes PQQ gleichzeitig die Funktionen des Mediators und die der Enzymkopplungsstelle. Hohe PQQOberflächenkonzentrationen führten bei Substratsättigung nach Optimierung der Elektrodenparameter $(\mathrm{pH}$, Pufferzusammensetzung) zu Stromdichten bis 200 pA/ $\mathrm{cm}^{2}$.

Die Anwendbarkeit der Sauerstoff-unabhängigen (PQQ)-GDH-MWCNT-Elektrode in einer enzymatischen Biobrennstoffzelle wurde durch die Kombination mit einer BOD-MWCNT-Elektrode demonstriert. Es entstand ein membranfreies System, welches Leistungsdichten im Bereich von 20-25 $\mu \mathrm{W} / \mathrm{cm}^{2}$ erreichen konnte. In dieser Kombination stellt die BOD-MWCNTElektrode nicht den limitierenden Faktor dar. Noch höhere Leistungsdichten können erreicht werden, wenn es gelingt den Elektrodenaufbau der (PQQ)-GDH-MWCNT-Elektrode weiter zu verbessern.

\section{Danksagung}

Für die finanzielle Unterstützung durch das MWFK Brandenburg (Projekt 3508-14/13) und das BMBF (Projekt PNT51513) möchten sich die Autoren bedanken. Gleicher Dank gilt unseren Kooperationspartnern Dr. von der Eltz und Dr. Meier von Roche Diagnostics (Penzberg) für die Bereitstellung der (PQQ)-GDH.

\section{Literaturverzeichnis}

Davis, F., Higson, S.P.J. (2007): Biofuel cells - Recent advances and applications. Biosens. Bioelectron. 22, 1224-1235.

Dokter, P., Frank, J., Jzn \& Duine, J.A. (1986): Purification and characterization of quinoprotein glucose dehydrogenase from Acinetobacter calcoaceticus L.M.D. 79.41. Biochem. J. 239, 163-167.

Gallaway, J.W., Barton, S.A.C. (2008): Kinetics of Redox Polymer-Mediated Enzyme Electrodes. J. Am. Chem. Soc. 130, 8527-8536.

Gao, F., Yan, YM., Su, L., Wang, L., Mao, LQ. (2007): An enzymatic glucose/O-2 biofuel cell: Preparation, characterization and performance in serum. Electrochem. Commun. 9, 989-996.

Goebel, G., Lisdat, F. (2008): Organic interlayers for oxygen reducing electrodes based on bilirubin oxidase and MWCNT modified gold. Electrochem. Commun. 10, 1691-1694.

Ivnitski, D., Atanassov, P., Apblett, C. (2007): Direct Bioelectrocatalysis of PQQ-Dependent Glucose Dehydrogenase. Electroanalysis 19, 1562-1568.

Katz, E., Willner, I. (2003): A biofuel cell with electrochemically switchable and tunable power output. J. Am. Chem. Soc.125, 6803-6813.

Kim, J., Jia, H., Wang, P. (2006). Challenges in biocatalysis for enzymebased biofuel cells. Biotechnol. Adv. 24, 296-308.

Kim, J., Parkey, J., Rhodes, C., Gonzalez-Martin, A. (2009): Development of a biofuel cell using glucose-oxidase- and bilirubin-oxidase-based electrodes. J. Solid State Electrochem. 13, 1043-1050.

Kuwahara, T., Homma, T., Kondo, M., Shimomura, M. (2009): Fabrication of enzyme electrodes with a polythiophene derivative and application of them to a glucose fuel cell. Synth. Met. 159, 1859-1864.

Laurinavièius, V., Razumienë, J., Kurtinaitienë, B., Gurevièienë, V., Marcinkevièienë, L., Bachmatova, I. (2003): Comparative characterization of soluble and membrane-bound PQQ-glucose dehydrogenases. Biologija (Vilnius). 2, 31-34.

Li, G., Xu, H., Huang, W., Wang, Y., Wu, Y., Parajuli, R. (2008): A pyrrole quinoline quinone glucose dehydrogenase biosensor based on screen-printed carbon paste electrodes modified by carbon nanotubes. Meas. Sci. Technol. 19: 065203-065210.

Lisdat, F., Wollenberger, U., Paeschke, M., Scheller, F.W. (1998): Sensitive catecholamine measurement using a monoenzymatic recycling system. Anal. Chim. Acta 368, 233-241.

Mao, F., Mano, N., Heller, A. (2003): Long Tethers Binding Redox Centers to Polymer Backbones Enhance Electron Transport in Enzyme »Wiring« Hydrogels. J. Am. Chem. Soc. 125, 4951-4957.

Murata, K., Kajiya, K., Nakamura, N., Ohno, H. (2009): Direct electrochemistry of bilirubin oxidase on three-dimensional gold nanoparticle electrodes and its application in a biofuel cell. Energ. Environ. Sci. 2, 1280-1285.

Otsuka, K., Sugihara, T., Tsujino, Y., Osakai, T., Tamiya, E. (2007): Electrochemical consideration on the optimum $\mathrm{pH}$ of bilirubin oxidase. Anal. Biochem. 370, 98-106.

Razumiene, J., Meskys, R., Gureviciene, V., Laurinavicius, V., Reshetova, M.D., Ryabov, A.D. (2000): 4-Ferrocenylphenol as an electron transfer mediator in PQQ-dependent alcohol and glucose dehydrogenase-catalyzed reactions. Electrochem. Commun. 2, 307-311.

Sakai, H., Nakagawa, T., Tokita, Y., Hatazawa, T., Ikeda, T., Tsujimura, S., Kano, K. (2009): A high-power glucose/oxygen biofuel cell operating under quiescent conditions. Energ. Environ. Sci. 2, 133-138. 
Schubert, K., Goebel, G., Lisdat, F. (2009): Bilirubin oxidase bound to multi-walled carbon nanotube-modified gold. Electrochim. Acta $54,3033-3038$

Stoica, L., Dimcheva, N., Ackermann, Y., Karnicka, K., Guschin, D.A., Kulesza, P.J., Rogalski, J., Haltrich, D., Ludwig, R., Gorton, L., Schuhmann, W. (2009): Membrane-Less Biofuel Cell Based on Cellobiose Dehydrogenase (Anode)/Laccase (Cathode) Wired via Specific Os-Redox Polymers. Fuel Cells 9, 53-62.

Stryer, L. (1995): Biochemistry, fourth ed. W.H. Freeman \& Company, New York.

Wang, S. C., Yang, F., Silva, M., Zarow, A., Wang, Y., Iqbal, Z. (2009): Membrane-less and mediator-free enzymatic biofuel cell using carbon nanotube/porous silicon electrodes. Electrochem. Commun. 11, 34-37.

Weigel, M.C., Tritscher, E., Lisdat, F. (2007): Direct electrochemical conversion of bilirubin oxidase at carbon nanotube-modified glassy carbon electrodes. Electrochem. Commun. 9, 689-693.

Willner, I., Katz, E., Patolsky, F., Bückmann, A.F. (1998): A biofuel cell based on glucose oxidase and microperoxidase-11 monolayerfunctionalized-electrodes. J. Chem. Soc. Perkin Trans. I 2, 1817 1822.

Willner, I., Yan, Y.-M., Willner, B., Tel-Vered, R. (2009): Integrated Enzyme-Based Biofuel Cells-A Review. Fuel Cells (Weinheim, Ger.) 1, 7-24.

Yang, W., Thordarson, P., Gooding, J.J., Ringer, S.P., Braet, F. (2007): Carbon nanotubes for biological and biomedical applications. Nanotechnology 18, 412001(12 pp.).

Ye, L., Haemmerle, M., Olsthoorn, A.J.J., Schuhmann, W., Schmidt, H.L., Duine, J.A., Heller, A. (1993): High current density »wired « quinoprotein glucose dehydrogenase electrode. Anal. Chem. 65, 238-241.

Yuhashi, N., Tomiyama, M., Okuda, J., Igarashi, S., Ikebukuro, K., Sode, K. (2005): Development of a novel glucose enzyme fuel cell system employing protein engineered PQQ glucose dehydrogenase. Biosens. Bioelectron. 20, 2145-2150.

Zayats, M., Katz, E., Baron, R., Willner, I. (2005): Reconstitution of Apo-Glucose Dehydrogenase on Pyrroloquinoline QuinoneFunctionalized Au Nanoparticles Yields an Electrically Contacted Biocatalyst. J. Am. Chem. Soc. 127, 12400-12406.

Zhao, H. Y., Zhou, H.M., Zhang, J.X., Zheng, W., Zheng, Y.F. (2009): Carbon nanotube-hydroxyapatite nanocomposite: A novel platform for glucose/O-2 biofuel cell. Biosens. Bioelectron. 25 , 463-468.

Zheng, W., Zhao, H., Zhou, H., Xu, X., Ding, M., Zheng., Y. (2010): Electrochemistry of bilirubin oxidase at carbon nanotubes. J. Solid State Electrochem. 14, 249-254.

\section{Autoren}

Christoph K. Tanne, M.Sc.

AG Biosystemtechnik

Technische Hochschule Wildau [FH]

Aktuelle Adresse: Institut für Mikrobiologie und Biotechnologie, Rhein. Friedrich-Wilhelms-Universität Bonn

chris.con.tanne@googlemail.com

Dipl.-Biotechnologe Gero Göbel

AG Biosystemtechnik

Technische Hochschule Wildau [FH]

gero.goebel@th-wildau.de

Prof. Dr. rer. nat. habil. Fred Lisdat

AG Biosystemtechnik

Technische Hochschule Wildau [FH]

fred.lisdat@th-wildau.de 